\title{
Erratum
}

\section{Erratum: Galinato et al., "Neurogenesis during Abstinence Is Necessary for Context-Driven Methamphetamine-Related Memory"}

In the article "Neurogenesis during Abstinence Is Necessary for Context-Driven Methamphetamine-Related Memory," by Melissa H. Galinato, Yoshio Takashima, McKenzie J. Fannon, Leon W. Quach, Roberto J. Morales Silva, Karthik K. Mysore, Michael J. Terranova, Rahul R. Dutta, Ryan W. Ostrom, Sucharita S. Somkuwar, and Chitra D. Mandyam, which appeared on pages 2029-2042 of the February 21, 2018, issue, Figure 6 appeared incorrectly.

The number of animals and the number of cells for electrophysiology studies have been reported incorrectly. The control group in Figure 6 for the electrophysiology experiment included data from $n=2$ male Wistar rats in addition to $n=2$ male GFAP-TK rats. The electrophysiology data for control rats between the two strains were not significantly different, and therefore were combined in Figure 6. The correct number of animals and cells for each condition are as follows: controls: $n=4$ rats, $n=13$ cells; TK-Valcyte rats: $n=3$ rats; $n=7-8$ cells; TK+Valcyte rats: $n=2$ rats, $n=15$ cells).

Additionally, the nonsignificant data in Figure $6 \mathrm{k}$ for the group TK-Valcyte is reported incorrectly. "Time to peak $224 \pm 28.0$ " should be "228 \pm 24.5 "; "peak amp $46.7 \pm 2.4$ " should be " $46.2 \pm 4.8$ "; "half-width $3.1 \pm 0.1$ " should be " $3.3 \pm 0.2$ "; "decay slope $-20.2 \pm$ 1.4 " should be " $-14.2 \pm 2.2$ "; "sAHP $1.1 \pm 0.3$ " should be " $0.4 \pm 0.5$ "; "rheobase $86.8 \pm 3.3$ " should be " $121 \pm 16.5$ "; and "spike threshold $-37.0 \pm 1.8$ " should be " $-38.9 \pm 2.9$ ".

The authors state, "These oversights did not materially affect the content of the paper and do not affect the conclusions of the paper as the statistical analyses were conducted on the correct number of cells in each group and were nonsignificant and remain nonsignificant”. The corrected figure and its legend appear below, and the online version has been corrected. Fig. 6

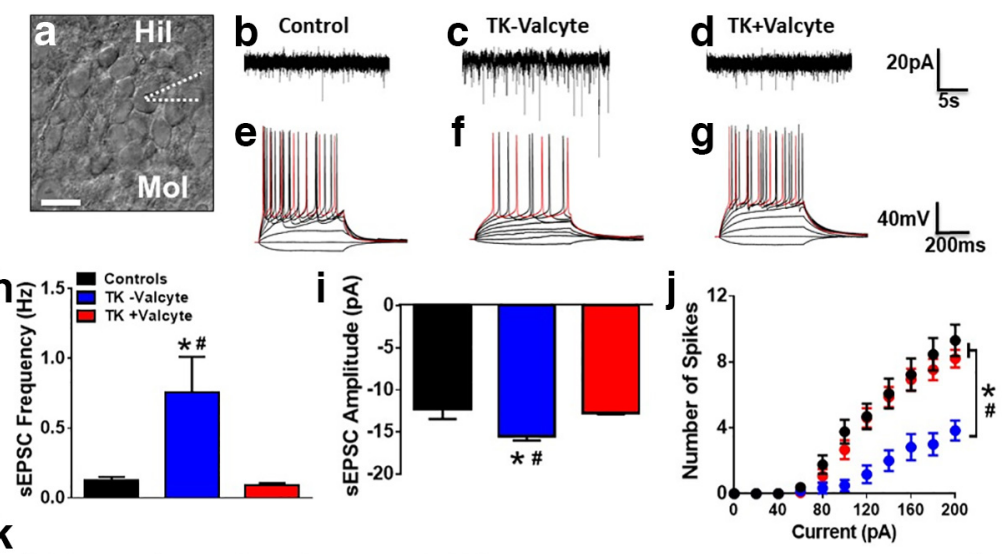

\begin{tabular}{|c|c|c|c|}
\hline \multicolumn{4}{|c|}{ Electrophysiological properties of DG neurons } \\
\hline & Control & TK-Valcyte & TK+Valcyte \\
\hline Time to peak (ms) & $217 \pm 24.0$ & $228 \pm 24.5$ & $290 \pm 10.6$ \\
\hline Resting potential (mV) & $-82 \pm 1.3$ & $-64 \pm 4.0 * \#$ & $-85 \pm 0.8$ \\
\hline Membrane resistance $(\mathrm{m} \Omega)$ & $553 \pm 70.8$ & $271 \pm 21.0 * \#$ & $597 \pm 47.8$ \\
\hline Rise slope to threshold (mV/ms) & $0.21 \pm 0.03$ & $0.20 \pm 0.02$ & $0.2 \pm 0.01$ \\
\hline Peak Amp (mV) & $43.1 \pm 2.0$ & $46.2 \pm 4.8$ & $53.9 \pm 0.9$ \\
\hline Half-width (ms) & $2.9 \pm 0.1$ & $3.3 \pm 0.2$ & $2.9 \pm 0.06$ \\
\hline Decay slope $(\mathrm{mV} / \mathrm{ms})$ & $-15.3 \pm 2.7$ & $-14.2 \pm 2.2$ & $-22.2 \pm 0.9$ \\
\hline sAHP $(\mathrm{mV})$ & $2.0 \pm 0.4$ & $0.4 \pm 0.5$ & $2.0 \pm 0.5$ \\
\hline Rheobase $(\mathrm{pA})$ & $71.6 \pm 3.9$ & $121.0 \pm 16.5$ & $91.7 \pm 7.2$ \\
\hline Spike threshold (mV) & $-36.9 \pm 1.4$ & $-38.9 \pm 2.9$ & $-37 \pm 0.7$ \\
\hline
\end{tabular}

Figure 6. $\boldsymbol{a}$, Representative image of the granule cell layer with GCNs and indication of the patch pipette on a selected neuron. Scale bar, $25 \mu \mathrm{m} . \boldsymbol{b}-\boldsymbol{d}$, Representative spontaneous EPSC (sEPSC) traces in voltage-clamp recording from control (b), TK-Valcyte $(\boldsymbol{c})$, and TK +Valcyte $(\boldsymbol{d})$ rats. $\boldsymbol{e}-\boldsymbol{g}$, Representative traces of action potentials elicited by depolarizing current injections. Traces were recorded from GCNs from control ( $\boldsymbol{e}$; drug and behavior naive $n=2$ male GFAP-TK rats and $n=2$ male Wistar rats; the electrophysiology data between the two strains were not significantly different and therefore were combined), TK-Valcyte $(\boldsymbol{f} ; n=3$ rats) and TK+Valcyte $(\boldsymbol{g} ; n=2$ rats) rats. $\boldsymbol{h}$, Frequency of sEPSCs in Hz from neurons. $\boldsymbol{i}$, Average of all amplitudes of all sEPSCs above $-10 \mathrm{pA}$ from GCNs. $\boldsymbol{j}$, Graphical relationship between the number of spikes elicited by increasing current injections in current-clamp recording. $\boldsymbol{k}$, Electrophysiological properties of GCNs from control, TK - Valcyte, and TK + Valcyte rats. Data are represented as the mean \pm SEM. Number of GCNs: $n=8-13$ controls, $n=7-8$ TK-Valcyte, and $n=15$ TK + Valcyte. ${ }^{*} p<0.05$ vs. controls and \# $p<$ 0.05 vs TK+Valcyte by ANOVA. 